\title{
Design and Characterization of the VMM1 ASIC for Micropattern Gas Detectors
}

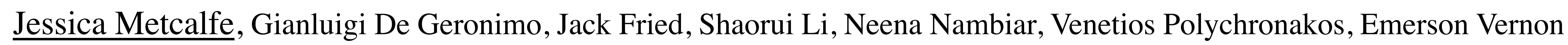

\section{BRDDKHOSTEN
NATIONAL LABORATORY}

\section{VMM1 ASIC}

The VMM1 ASIC was designed for use with Micromegas and Thin Gap Chambers (TGCs) for ATLAS Upgrade. It features a range of gains and peaking times to allow use with other types of micropattern gas detectors as well. It incorporates several innovative features to allow operation in a fast trigger mode in the ATLAS Level 1 trigger as well as in a time projection mode ( $\mu$-TPC mode) for precision track reconstruction. The VMM1 also features smart token passing reading out only those channels above threshold and the first-order neighbors in order to reduce the bandwidth and increase the potential for new physics.


Figure 1: Left: Diagram of a Micromegas Detector slated for part of the New Small Wheel in the ATLAS Upgrade that will replace the Cathode Strip Chapmbers (CSCs) shown on the right. Right: The current ATLAS Detector.

\section{VMM1 Design}

The VMM1 ASIC was fabricated in a commercial $130 \mathrm{~nm}$ CMOS from IBM. It has 64 front-end channels each consisting of low noise charge amplifier (CA), shaper with baseline stabilizer (SA), a discriminator with trimmer (DSC), a peak- and time-detector (PD/TD), a time-to-amplitude converter (TAC), multiplexing with smart token, and direct Time-over-Threshold outputs (TOT) as shown in the figure below.

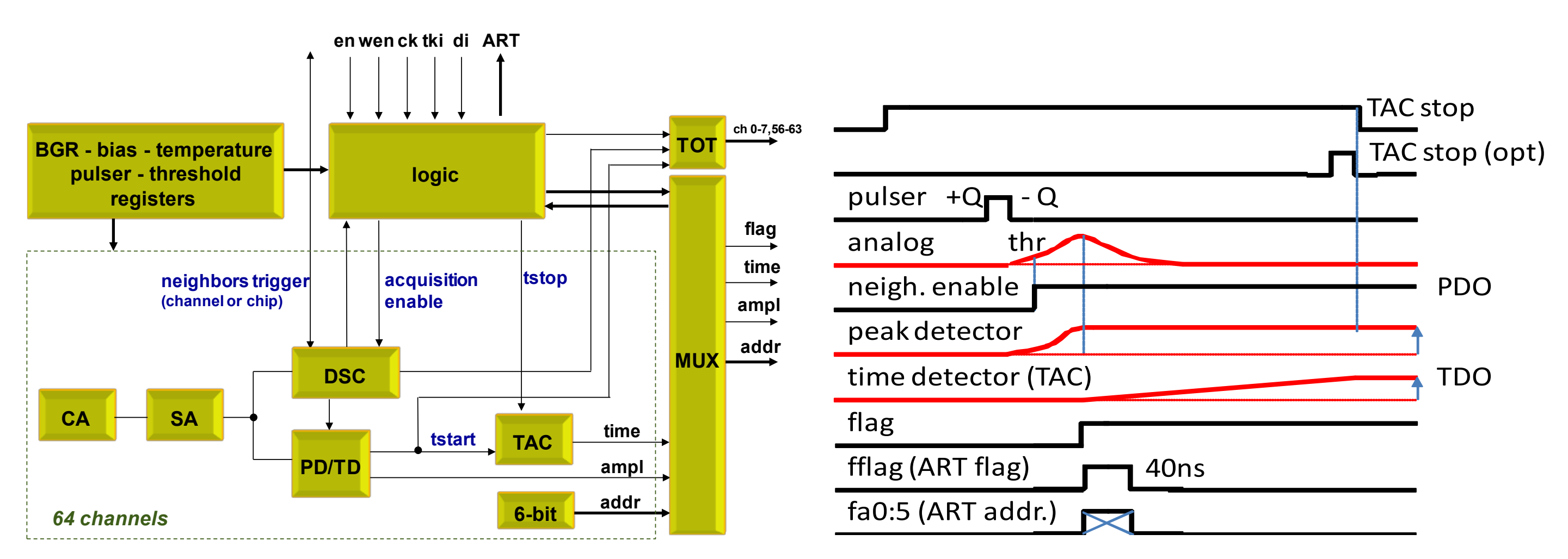

Figure 2: Left: VMM1 layout. Right: VMM1 Signal Acquisition.

\section{Flexible Functionality of the VMM1}

- Selectable Gain

- The nominal gain value has the option of $0.5,1,3$, and $9 \mathrm{mV} / \mathrm{fC}$ to

accomoctable Peaking Time

- The Peaking Time or integration time can be selected from the following options: $25 \mathrm{~ns}, 50 \mathrm{~ns}, 100 \mathrm{~ns}$, and $200 \mathrm{~ns}$.

-Adjustable TAC slope

The time-amplitude-conversion (TAC) slope can be adjusted according to $125 \mathrm{~ns} / \mathrm{V}, 250 \mathrm{~ns} / \mathrm{V}, 500 \mathrm{~ns} / \mathrm{V}$ and $1 \mu \mathrm{s} / \mathrm{V}$.

Neighbor Readout

- There is an option to read out the 2 neighboring channels of a channel with a signal over threshold. In the case the neighbor channel is read ou by a neighboring VMM1, the information is passed the Smart Token Passing

- This feature allows only the signals above threshold to be read out reducing bandwidth requirements.

Fast Trigger Readout

- The Address in Real Time (ART) reads out the address of the first

channel with a signal hit for fast trigger applications

- Timing Mode

- The Time-over-Threshold (ToT) and the Time-to-Peak (TtP) can be read out of the analogue monitor for the first and last eight channels.

- Threshold Trimming

- The threshold of each channel may be trimmed by approximately $15 \mathrm{mV}$ in order to equalize channel-to-channel variation.

Sub-Hysteresis

- The fast comparator introduces a hysteresis on the threshold of approximately $\pm 10 \mathrm{mV}$. The Sub-Hysteresis counteracts this by adjusting section.)

Internal Pulser

- An internal pulser was incorporated into the VMM1 for testing purposes.

VMM1 ASIC flexible enough to work with several applications: Micromegas (Micro-Mesh Gaseous Detector) Thin Gap Chambers

Gas Electron Multiplier Detectors

in both precision tracking and fast trigger modes

with simulated charge resolution of $\sim 5000 \mathrm{e}^{-}$at $25 \mathrm{~ns}$ and $200 \mathrm{pF}$ and timing resolution $1 \mathrm{~ns}$ at $1 \mathrm{~V}$ signal amplitude

\section{$\underline{\text { VMM1 Features }}$}

\section{Peak Amplitude}

The peak detector measures the amplitude of the signal peak very precisely. The output amplitude, called the PDO (Peak Detector Output), measures the signal

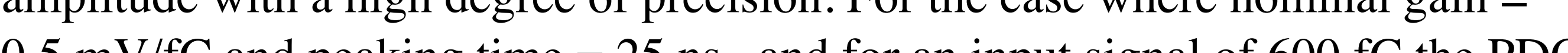

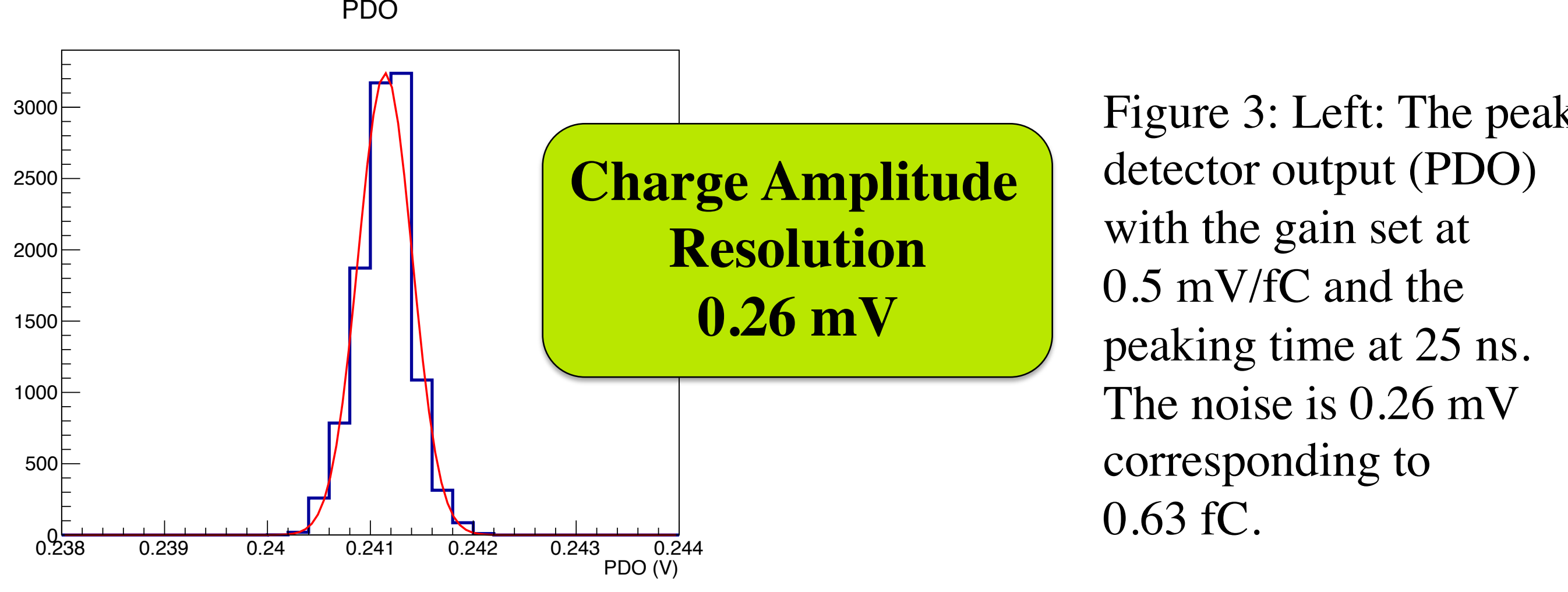

There are 4 nominal values each for gain and peaking time. The combination of the configuration determines the real signal gain. The figure below shows the gain linearity for each of the gain settings (upper plot). The bottom plot shows the percentage of the linearity error or the percentage error from the expected value from the fit. The linearity is of the order of less than $0.1 \%$.
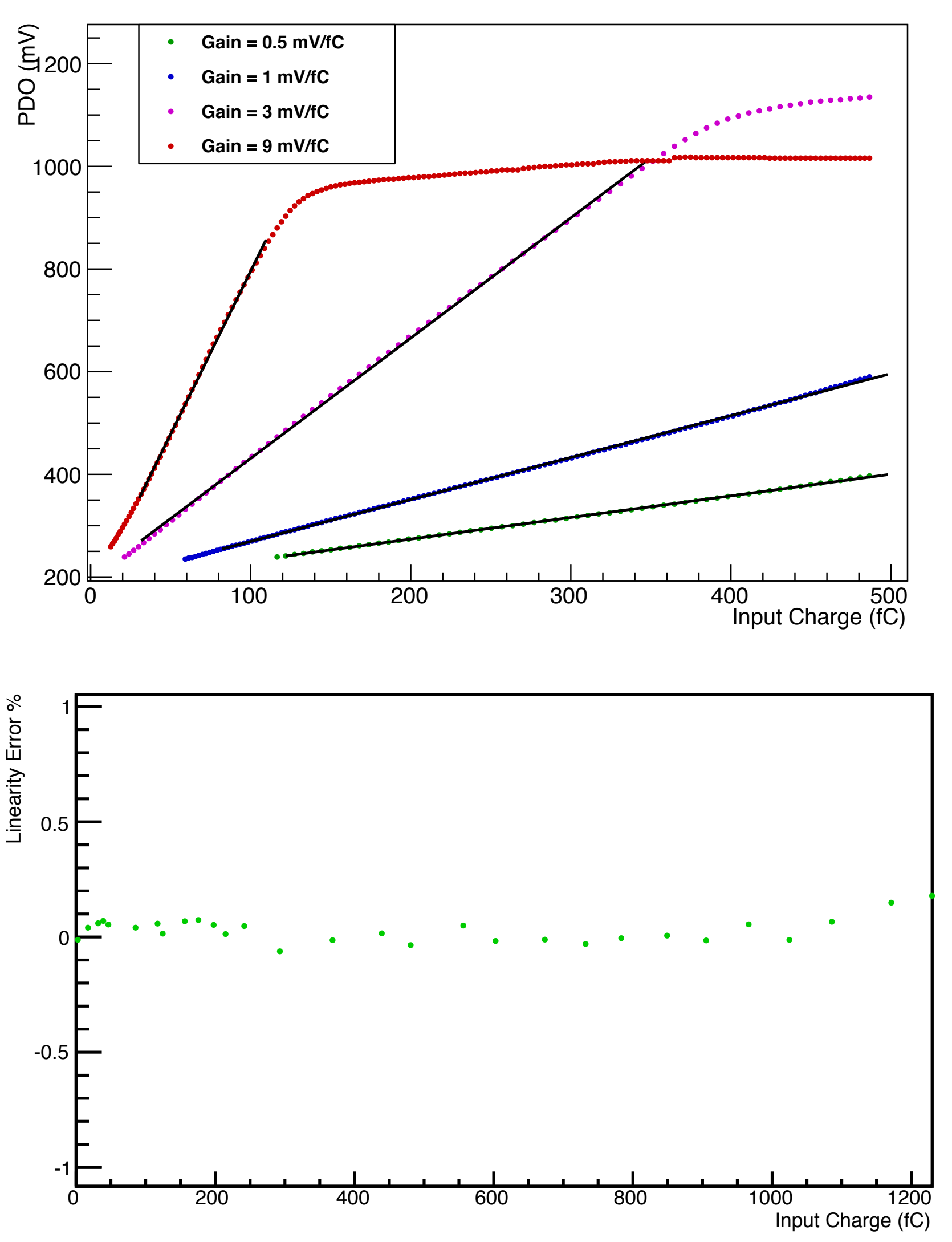

Figure 4: Top: PDO response as a function of input charge for each of the nominal gain settings and fits to extract measured gains. Bottom: The percentage linearity error or residual percentage of the PDO response with an external pulser taken at nominal gain of $1 \mathrm{mV} / \mathrm{fC}$ and peaking time of $50 \mathrm{~ns}$.

\section{Time Detection}

The time is measured by charging a capacitor at a specific rate given by the Time-Amplitude-Conversion (TAC) slope from the time the peak is detected until the signal is read out as shown in Figure 2. The TAC slope has several settings to accommodate different applications depending on the signal integration time. In the figure below on the left the TDO response shows a
timing resolution of $0.04 \mathrm{~ns}$ for nominal gain $=0.5 \mathrm{mV} / \mathrm{fC}$, peaking time timing resolution of $0.04 \mathrm{~ns}$ for
$=25 \mathrm{~ns}$, and TAC $=125 \mathrm{~ns} / \mathrm{V}$.
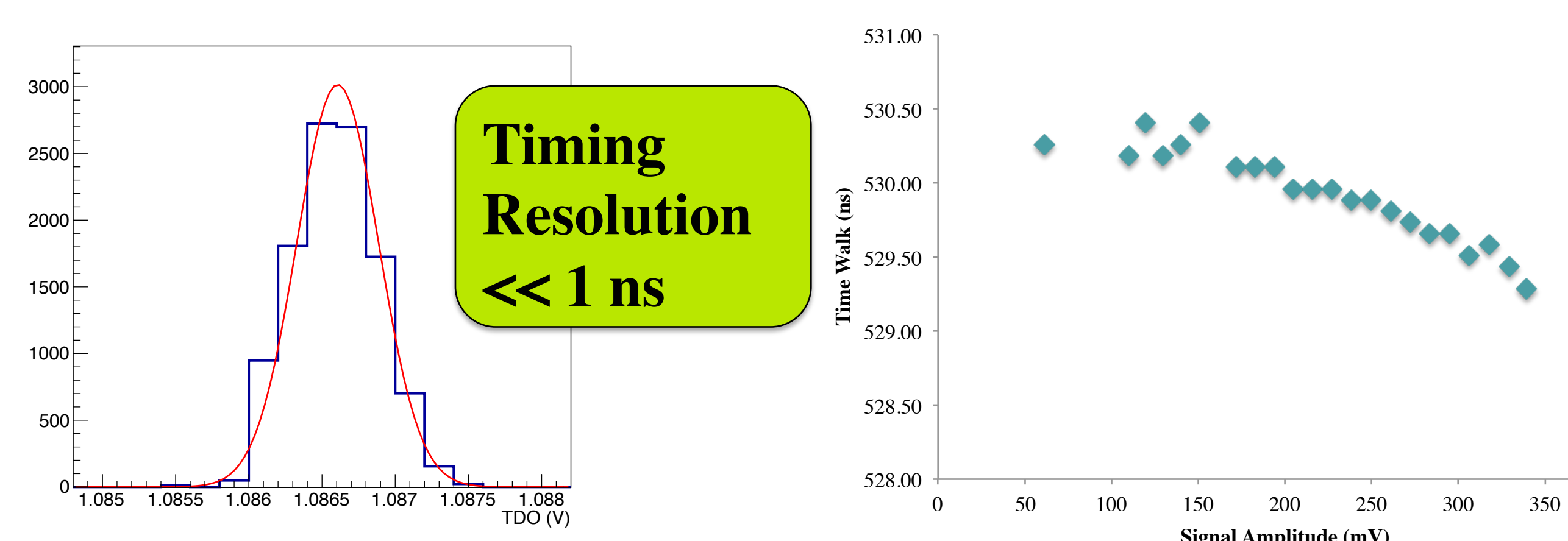

Figure 5: Left: Timing (TDO) resolution for gain $=0.5 \mathrm{mV} / \mathrm{fC}$ and peaking time $=25 \mathrm{~ns}, \mathrm{TAC}=1 \mu \mathrm{s} / \mathrm{V}$. The resolution is $0.28 \mathrm{mV}=0.04 \mathrm{~ns}$. Right: Time Walk at $\mathrm{g}=1, \mathrm{pT}=25 \mathrm{~ns}$ shows approximately $1 \mathrm{~ns}$ variation over the range of signal amplitudes.

The time-walk measures the jitter of the time the peak is detected as the signal amplitude is varied. Ideally, the time is independent of the signal amplitude. In the figure on the left the time-walk is shown for the VMM1 and of the order of $1 \mathrm{~ns}$

\section{Time Over Threshold \& Time To Peak}

The first version of the VMM has timing output on 16 channels. There are two modes of operation Time-over-Threshold (ToT) and Time-to-Peak (TtP).

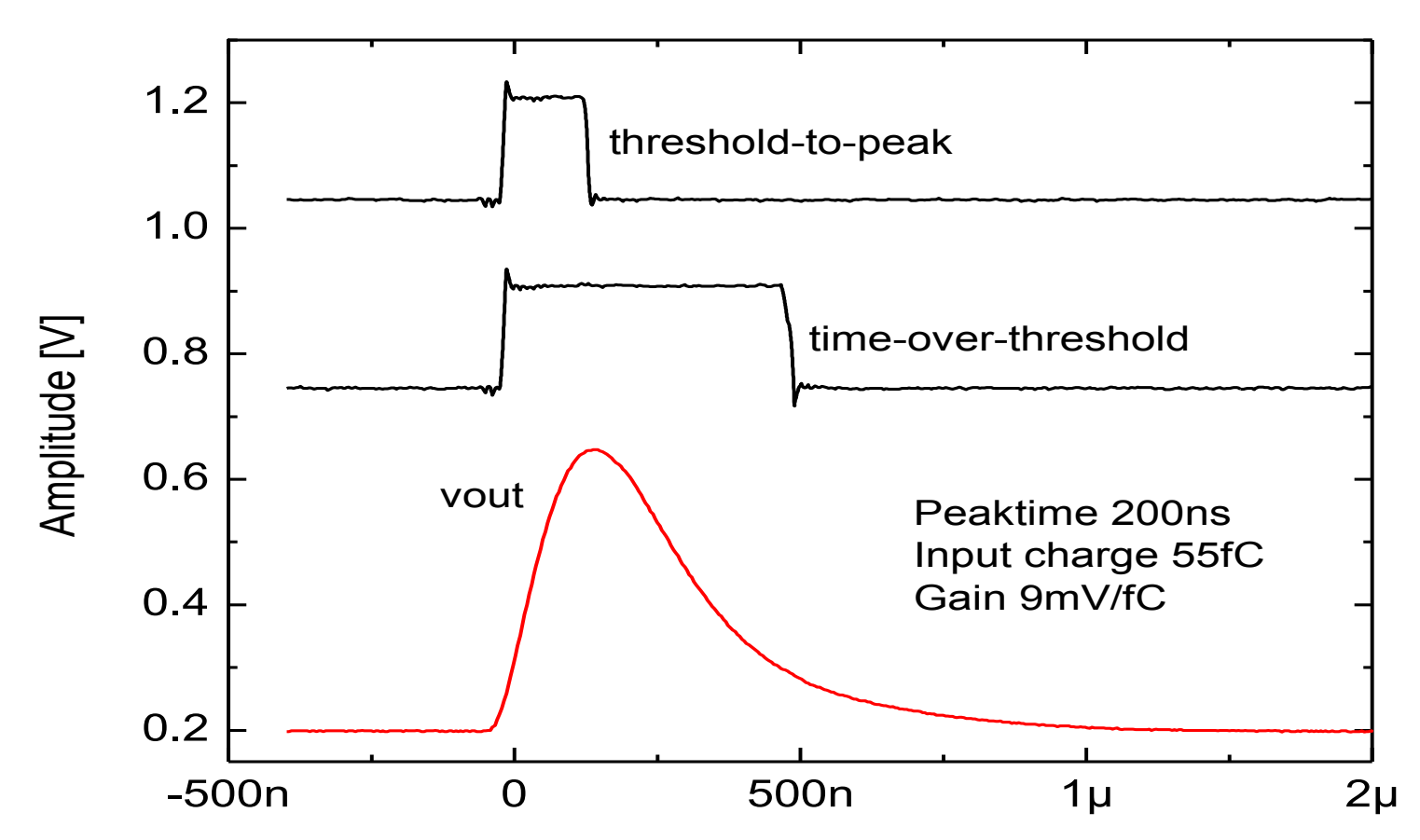

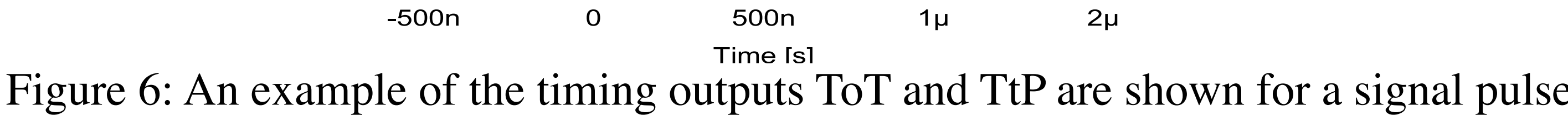

\section{VMM1 Features}

\section{Threshold}

The threshold inherently has some channel to channel variation. A threshold trim was included in the VMM1 design to equalize thresholds by allowing each individual channel to be trimmed by approximately $0-15 \mathrm{mV}$. The percent of signals that were returned by the acquisition at each input amplitude resulting in an S-curve or turn-on curve. The mid-point of the turn-on gives the resulting in an S-curve or turn-on curve. The mid-point of the turn-on gives
threshold for a particular channel. The figure below on the left shows the variation in the threshold channel by channel. The plot to the right demonstrates the range of the trim for 5 channels. In version 1 of the VMM the demonstrates the range of the trim for 5 channels. In version 1 of the VMM the
trim range is not sufficient to equalize all channels, but will be corrected in the second version.

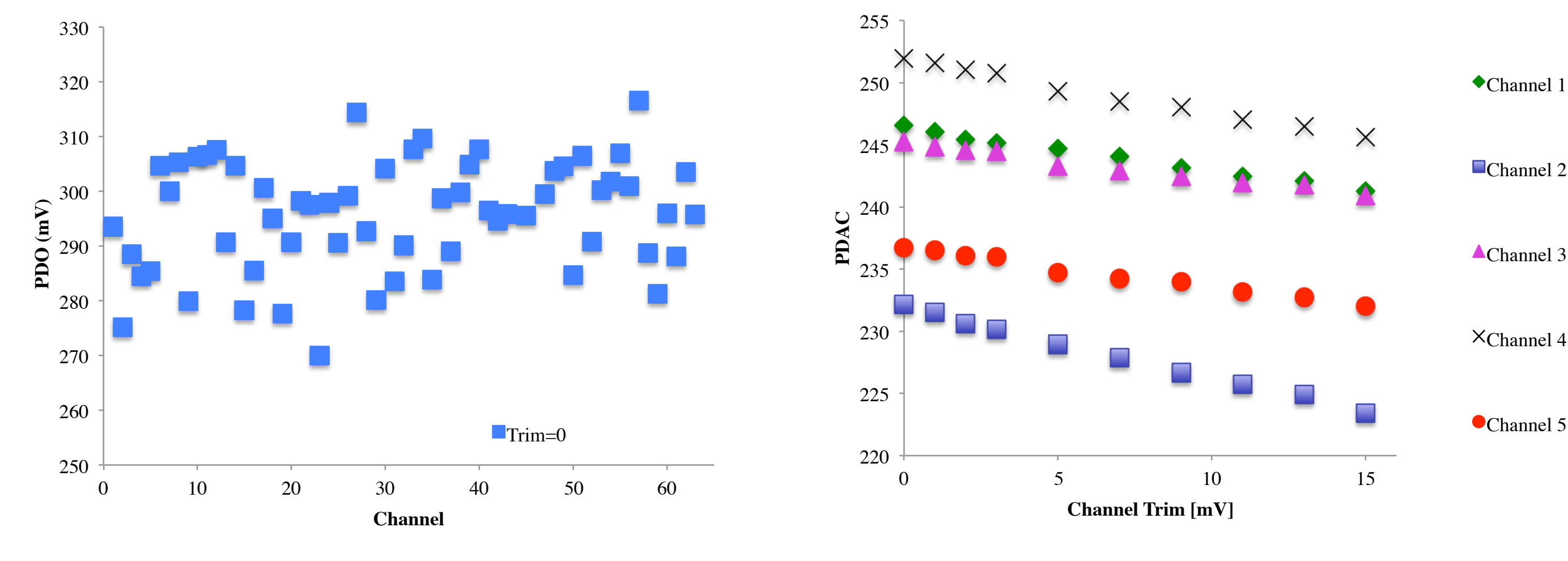

Figure 7: Left: Thresholds extracted from S-curve measurements versus channel number for the minimum trim setting equal to $0 \mathrm{mV}$. Right: The trim allowance is shown for each programmable trim setting for 5 channels.

\section{$\underline{\text { Sub-Hysteresis }}$}

The fast comparator introduces a hysteresis, which effectively raises the minimum threshold by approximately $10 \mathrm{mV}$. The sub-hysteresis was introduced to compensate for this effect by moving the threshold level so that it triggers close to the baseline and resets after the peak is detected. See the diagram in Figure 8 .



Figure 8: Diagram of the sub-hysteresis operation.

Figure 9 shows signal amplitude versus the input charge with and without the sub-hysteresis function for the lowest working threshold. Without the subhysteresis function the lowest threshold is at the baseline. With the subhysteresis function engaged, the threshold can be tuned down even further to a nominal threshold (not including the hysteresis) $9 \mathrm{mV}$ below the baseline. The PDO is able to read out input amplitudes $56 \mathrm{fC}$ smaller than without for gain $=$ $0.5 \mathrm{mV} / \mathrm{fC}$, peaking time $=25 \mathrm{~ns}$, and input capacitance equal to $2.44 \mathrm{pF}$. The measurements show that for these conditions the VMM1 can measure signal amplitudes $11 \mathrm{mV}$ smaller with the Sub-Hysteresis feature than without.

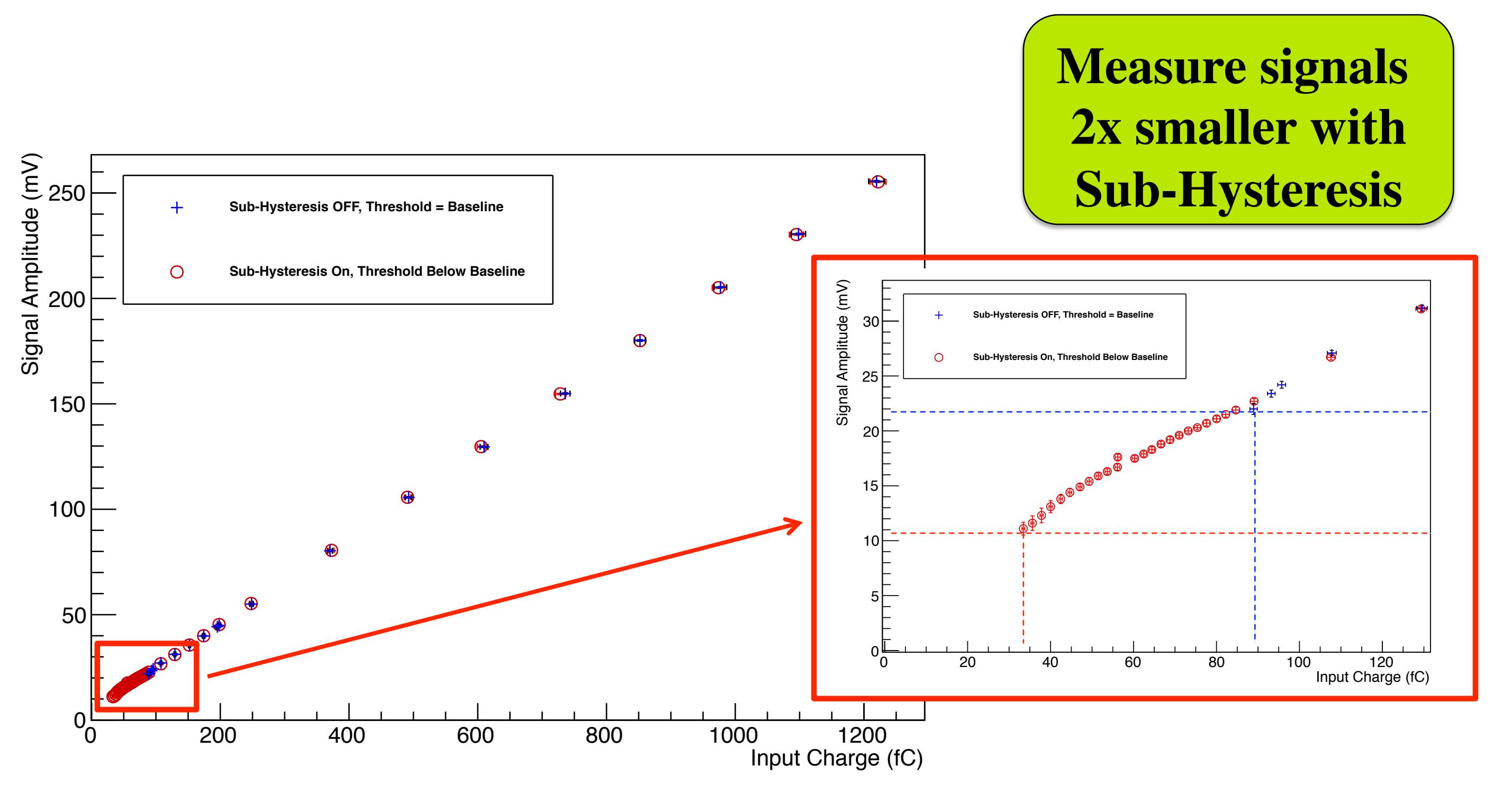

Figure 9: Left: The lowest threshold setting without the Sub-Hysteresis and the lowest threshold with the Sub-Hysteresis engaged is shown as a function of the input charge. Right. When the Sub-Hysteresis is on, he threshold can be set lower-below the baseline and much smaller charges can be read out.

\section{Conclusion:}

- The first version of the VMM was very successful! - charge resolution $0.26 \mathrm{mV}$ at nominal gain $=0.5 \mathrm{mV} / \mathrm{fC}$ - timing resolution $<<1 \mathrm{~ns}$ - measure signals 2x smaller with Sub-Hysteresis feature - VMM2 will improve upon the first edition and add new features such as digitization and more timing outputs 